\title{
Ad Hoc Reviewers (1992)
}

Bamford, J.

Jeanrenaud, X.

Mokri, B.

Barth, A.

Johansson, B.

Moulin, Th.

de Merloose, $\mathrm{P}$.

Kappelle, L.-J.

Muller, H.R.

de Tribolet, N.

Kaste, $\mathrm{M}$.

Niederkorn, K.

Diener, H.C.

Kiessling, $\mathrm{M}$.

Paulson, O.

Grotta, J.

Koudstall, P.

Pierrot-Deseilligny, Ch.

Hacke, W.

Kuntzer, Th.

Rautenberg, W.

Halliday, A.M.

Kunze, S.

Riitishauser, W.

Hart,R.

Kuwert, T.

Schwartz, A.

Hass, W.

Maeder, Th.

Steinke, W.

Henn, V.

Marshall, J.

Strauer, B.

Homberg, V. 
Marx, P.

von Reutern, G.

Hornig, C.

Mattle, $\mathrm{H}$.

Widder, B.

Jallon, P.

Meienberg, $\mathrm{O}$.

Janzer, R.

Mohr, J.P. 